\title{
USE OF THE ADAMO DATA MANAGEMENT SYSTEM WITHIN ALEPH
}

\author{
Z. QIAN, S. TANG, W. ZHAO * \\ IHEP, Academica Sinica, Beijing, China \\ S.M. FISHER *, J. HARVEY \\ Rutherford Appleton Laboratory, Didcot, $G B$ \\ M. BOANO, J. BUNN, R. McCLATCHEY, V. EMILIANI, P. PALAZZI * \\ CERN, Geneva, Switzerland
}

R. BRAZIOLI *, A. PUTZER

IHEP, Heidelberg University, Fed. Rep. Germany

M.G. GREEN

Royal Holloway College, Univ. of London, GB

and R. FANTECHI

Instituto di Fisica, Pisa, Italy

\begin{abstract}
ADAMO is a data management system for defining data and manipulating them from FORTRAN programs. A form of the Entity-Relationship (ER) model and the data flow diagrams of structured analysis have been combined to provide a system suited to algorithmic work. The FORTRAN interface consists of calls to a subroutine package with a few data manipulation primitives. Data items are visible in variables carrying meaningful names; the code is compact and readable. The ER data structures, mapped onto tables, are compatible with a relational data base management system. Tools have been written to copy tables to and from a relational data base. The system has been used for several applications in the fields of event simulation and reconstruction, online systems, detector description, support tools and packages.
\end{abstract}

\section{Introduction}

The Aleph DAta MOdel (ADAMO) system was developed as an independent project within the ALEPH ${ }^{+}$collaboration, its aim being to cope with the complexity of data in a modern particle physics experiment. It has been stated that

$$
\begin{aligned}
\text { PROGRAMS }= & \text { ALGORITHMS } \\
& + \text { DATA STRUCTURES, }
\end{aligned}
$$

and in the past much attention has been devoted to the algorithms. Now that detectors are larger

\footnotetext{
* Denotes those who are also authors of the ADAMO system.

+ ALEPH is an experiment at the LEP collider at CERN.
}

and more complex, data structures and data management have become increasingly important. The design of ADAMO has been influenced by clear trends in the software industry, such as the prominence of relational data bases, increasing use of engineering practices, data abstraction and the importance of a programming environment with adequate software development tools.

In defining and constructing the system, it was decided to distinguish between the INTERNAL (machine) and EXTERNAL (programmer, user) view of the data, defining a CONCEPTUAL view to which they can both refer [1]. To achieve this a DATA MODEL was adopted, with a set of data structures, manipulation operators and validation

0010-4655/87/\$03.50 @ Elsevier Science Publishers B.V.

(North-Holland Physics Publishing Division) 
primitives. A machine readable DATA DICTIONARY, accessible to all the programs related to a given application, is the physical support of the model. Finally, it was considered essential that data elements be visible in the final code as variables carrying meaningful names, while manipulation operators would be seen as calls to a subroutine package.

\section{Modelling data}

For data to be useful in providing information, they have to be organized so that they represent the real world as closely as possible, and that, at the same time, they can be easily handled by computers. These two requirements are often conflicting. To determine how best to arrange data for each application it is convenient to formulate general statements about how data are organized and processed in all applications. A consistent, formal set of such statements defines a DATA MODEL [2].

A data model is a combination of at least three components [3]:

1. a collection of data structure types;

2. a collection of operators which can be applied to any valid instance of the data types listed in (1), to retrieve, derive or modify data from any part of those structures in any combination desired;

3. a collection of general integrity rules, which define the set of consistent database states - these rules are general in the sense that they apply to any database using the model.

After several attempts to model typical particle physics applications, the Entity-Relationship (ER) model was selected. It was originally proposed by Chen in 1976 [4] as a way to unify the network and relational database approaches, and in the last decade there has been a proliferation- of theoretical extensions and practical applications of it. The model is now part of both the ACM and IEEE recommended curricula. It is simple enough that the basic concepts can be learned readily, yet sufficiently powerful for scientific and technical applications. It adopts the view that the real world consists of ENTITIES, things that can be dis- tinctly identified and have a fixed number of characteristics called ATTRIBUTES, and RELATIONSHIPS, that are associations among entities. An important feature of the model is the ER diagram, a graphical representation of the data with boxes and arrows. These diagrams, together with data flow and state transition diagrams (which are complementary models of a system), form a compact description that is easy to grasp and convenient for discussions.

\section{Programming with ADAMO}

The analyst/programmer sees ADAMO as a set of data design and program preparation tools, integrated in the command environment of the operating system (VMS and VM at present). He then programs his application in FORTRAN using the data manipulation subroutines of the TAble Package (TAP). The ADAMO system, including the overall system architecture [5], and a complete example, from modelling to a working program [6] is described in a series of notes.

The tools provide a complete path from the ER diagrams, through the data structures (entities, relationships and attributes) described in the Data Definition Language (DDL) and mapped onto tables, down to the FORTRAN variables to which those data correspond. Other tools are available to validate and draw data flow diagrams of Structured Analysis (SA) [7], to document the data structures, to define the schema of a relational data base and transfer data to and from it. Tools can be activated individually with a command, and communicate asynchronously through interface files. Online documentation is available via HELP files that correspond to the printed documents, and templates for the VMS Language Sensitive Editor (LSE) assist with the syntax and the semantics of the DDL.

After successfully running the data definition and program preparation tools, the application program is coded, including calls to the data manipulation subroutines of the TAP (LSE templates are also available for this purpose). The TAP consists of about 75 subroutines (although a typical application uses about twenty), among 
them the essential data manipulation primitives of the model:

\section{INSERT, REPLACE, DELETE, COUNT, SELECT, FETCH, RELATE, NAVIGATE}

Subroutines exist to check the validity of various aspects of the data; for example that attributes of entities are within the range specified in the DDL.

The TAP normally makes a number of checks on the consistency between the subroutine parameters and the data structure, but these checks may be inhibited in production programs to save time.

Other subroutines copy tables to and from a Direct Access File (DAF) where they are identified by a multi-level key. As all data are held in tables, building an interface to a Relational DBMS was straightforward. This interface tool, the TOR is able to copy a set of ADAMO tables from a DAF to an ORACLE [8] data base, and back again. The ORACLE RDBMS provides facilities such as multi-user access and consistency control, and is supported by sophisticated database management tools, but it is not always convenient to use it directly from an application program. There are three basic reasons for this:

- ORACLE is very slow when compared with the TAP;

- Coding with the TAP is much easier than using the query language interface provided by ORACLE;

- Not many sites have ORACLE installed.

\section{An illustration of using the ADAMO system}

This section illustrates the steps performed by the application programmer using ADAMO, to create a section of the ALEPH reconstruction program: cluster finding in the electromagnetic calorimeter (ECAL). In developing this code the techniques of SASD were used in conjunction with ADAMO. The ADAMO aspects are stressed here, as the use of the SASD technique by ALEPH is reported in another submission to this conference [9]. This work is described in more detail in one of the ADAMO application notes [10]. A complete list of such notes may be found in ref. [11].
The calorimeter is read out via cathode pads. The pads are grouped together to reduce the number of output channels to 218000 . Each group is known as a storey.

One of the dataflow diagrams (DFD) describing the reconstruction of the cluster energies is shown in fig. 1a. In contrast to this diagram, which shows how the data are processed, the ER diagram of fig. 2 defines the structure of the data as entities and the relationships between them. It was found essential to develop the two types of diagram in parallel and iteratively, as it is is hard to imagine how to process data, if the data are not understood; and it is not possible to know what data are needed until the processing requirements are clear.

Corresponding to each ER diagram is a formal description of that diagram in the Data Definition Language (DDL) of ADAMO. An extract from the ECALData DDL showing definitions of some of the entity sets (ESET), relationships (RSET) and attributes is presented in fig. 3. DDL files are processed by an ADAMO tool to produce an internal storage representation called the ADAMO Data Dictionary (ADD). To provide a real link between data flow and ER diagrams, data flows are ultimately expanded down to components which are entity sets, attributes and relationships. As this is described in the DDL (fig. 3), the data flow plotting tool is able to redraw the diagram where the data flow lines are annotated with the expanded names as illustrated in fig. $1 \mathrm{~b}$.

Each entity set has an attribute (ID) to hold a unique identifier for each entity. Within the application program each entity set is materialised as a table. Each entity gives rise to a row in the corresponding table with the columns containing the values of the attributes of that entity. In this table each attribute has a corresponding column as does each relationship for which the arrow on the ER diagram points away from the entity set. Thus the entity set ESDA has four attribute columns as listed in the DDL of fig. 3 and three relationship columns as shown in the ER diagram of fig. $2 b$ and the RSET definitions of the DDL.

The contents of the data dictionary can be listed in tabular form. Fig. 4 shows an extract from one of the several listings which can be 


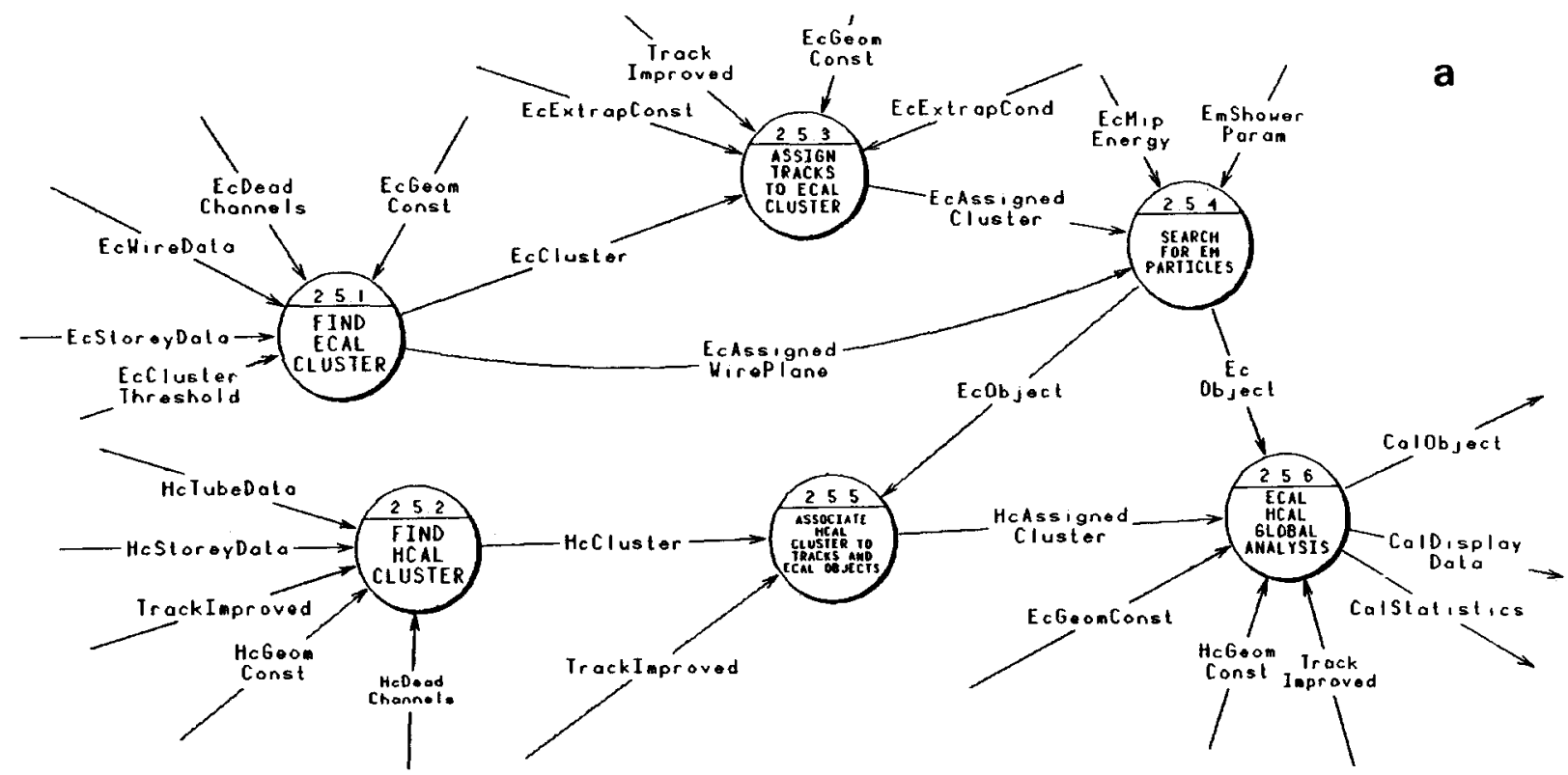

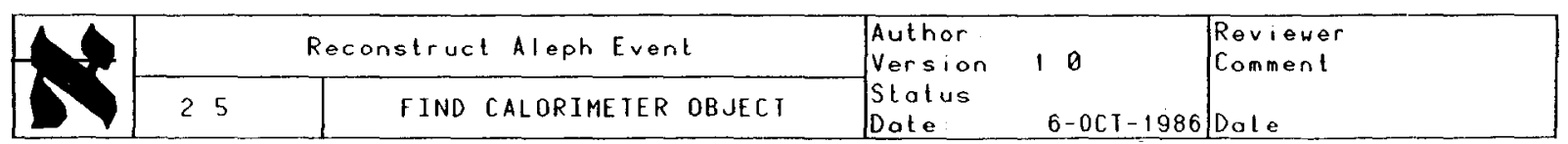

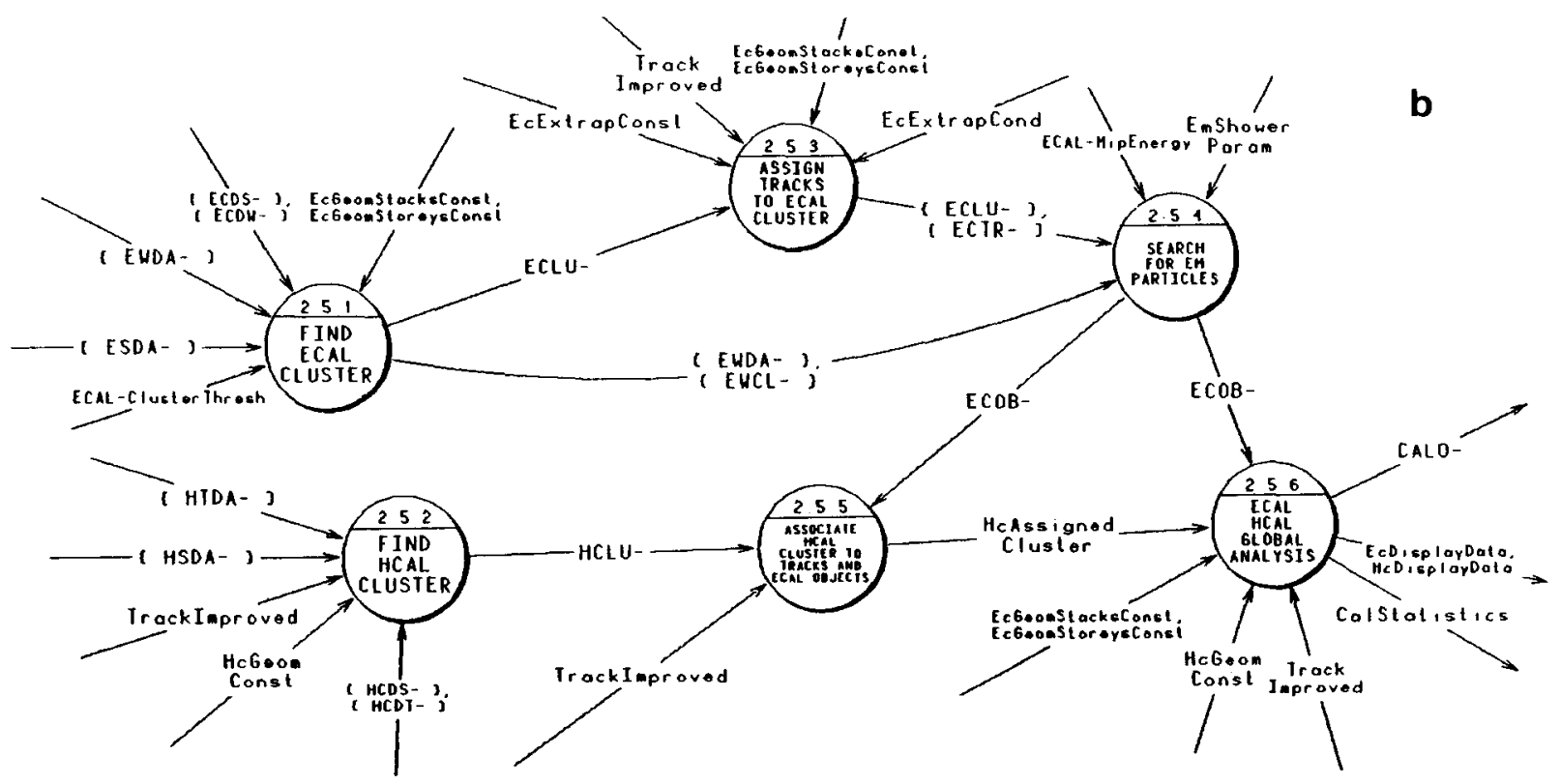

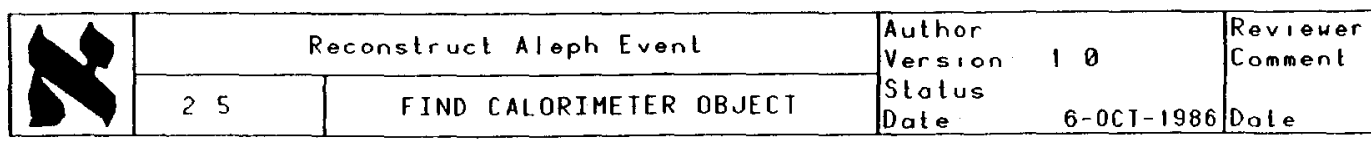

Fig. 1. (a) ECAL Dataflow diagram 2.5 (unexpanded); (b) ECAL dataflow diagram 2.5 (expanded). 


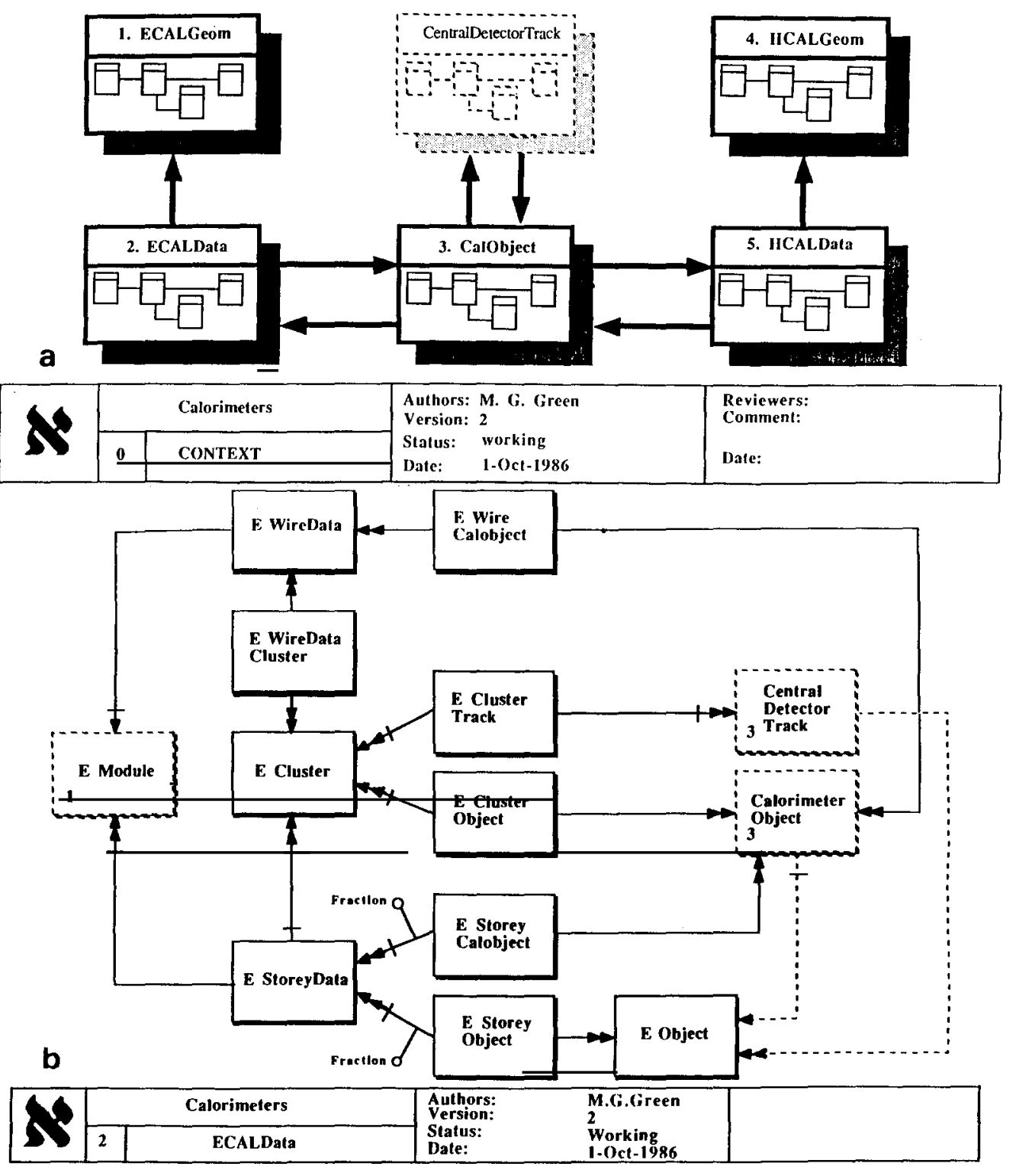

Fig. 2. (a) ECAL ER diagram - CONTEXT; (b) ECAL ER diagram - ECALData.

produced. This output forms an important part of the documentation of the application program.

The next stage is to pass the ADD through the FORTRAN Language Binder to produce a table initialisation routine and FORTRAN COMMON blocks and declaration statements for insertion into the application program. The variable names may be either "VAX-style", formed by concatenating the entity set and attribute names to give variables such as ESDA_ Row, or ANSI six character names such as ESDARW.

An example of a routine from the ECAL reconstruction is shown in fig. 5. This particular routine collects into a cluster all storeys that are connected by at least one corner. This its principal input is the table ESDA, and its output is the table ECLU. Calls to TAP routines are underlined in this listing and illustrate typical operations on 


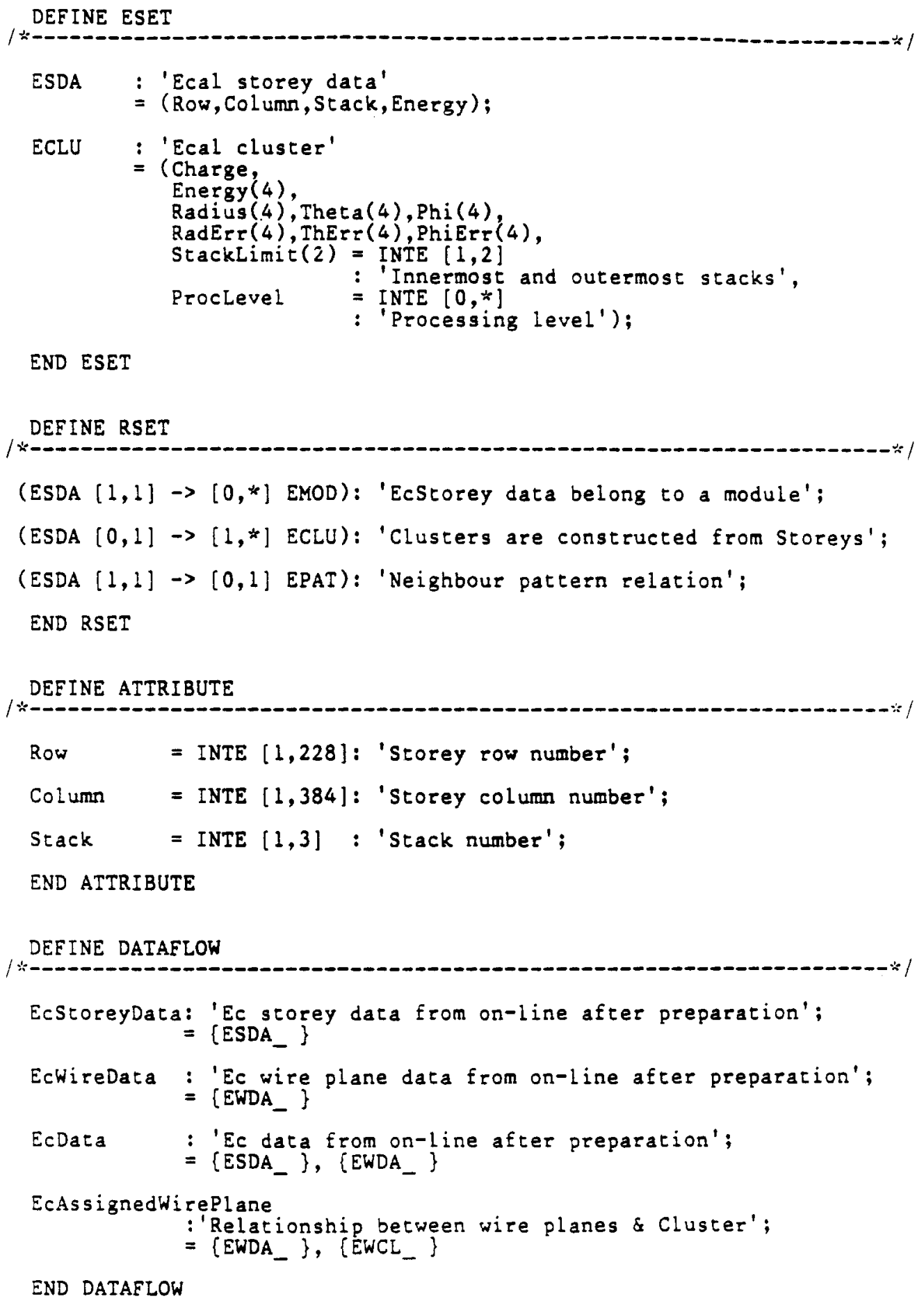

Fig. 3. Extract from DDL for ECALData. 
the tables such as fetching a row (FETTAB and GETTAB), insert a new row (INSENT) and inserting an entry into a relationship column (INSREL). The table ESDA for a typical simulated event is shown in fig. 6 .

\section{Applications}

\subsection{Reconstruction and simulation programs}

The previous section described how ADAMO is being used in the reconstruction program. Work was also performed to test the suitability of

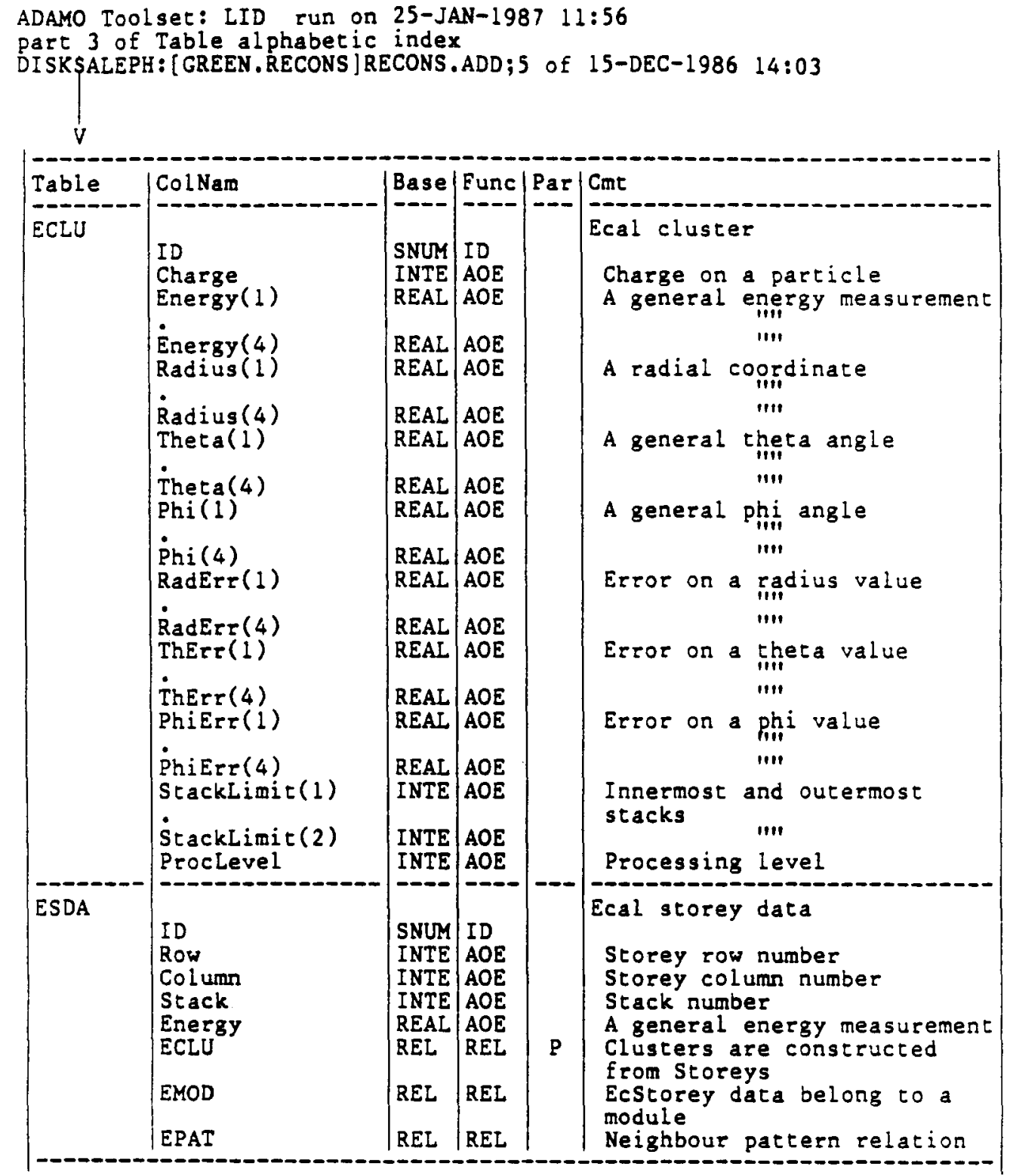

Fig. 4. Extract from ADD listing. 


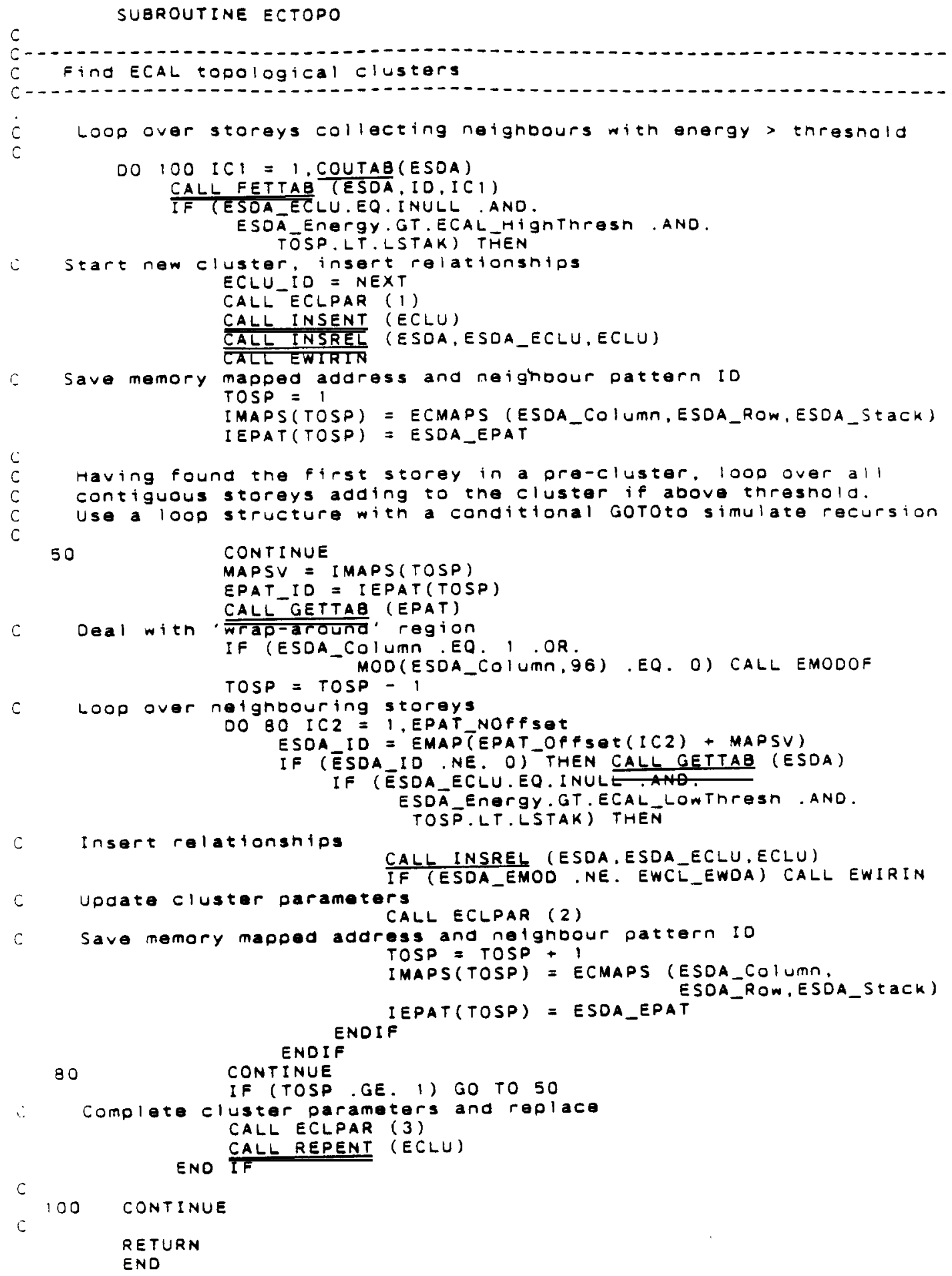

Fig. 5. An example for ECAL code using the TAP. 
ADAMO and the TAP in the ALEPH simulation program, by converting a part of it to use the TAP, and comparing it with the original [12]. The original program has been written using direct calls to a memory manager, where each person working on the program had used his favourite data structures.

Taking as input existing code and data descriptions, the system was modelled in a way which reflected the existing data structure such that the

Ecal storey data

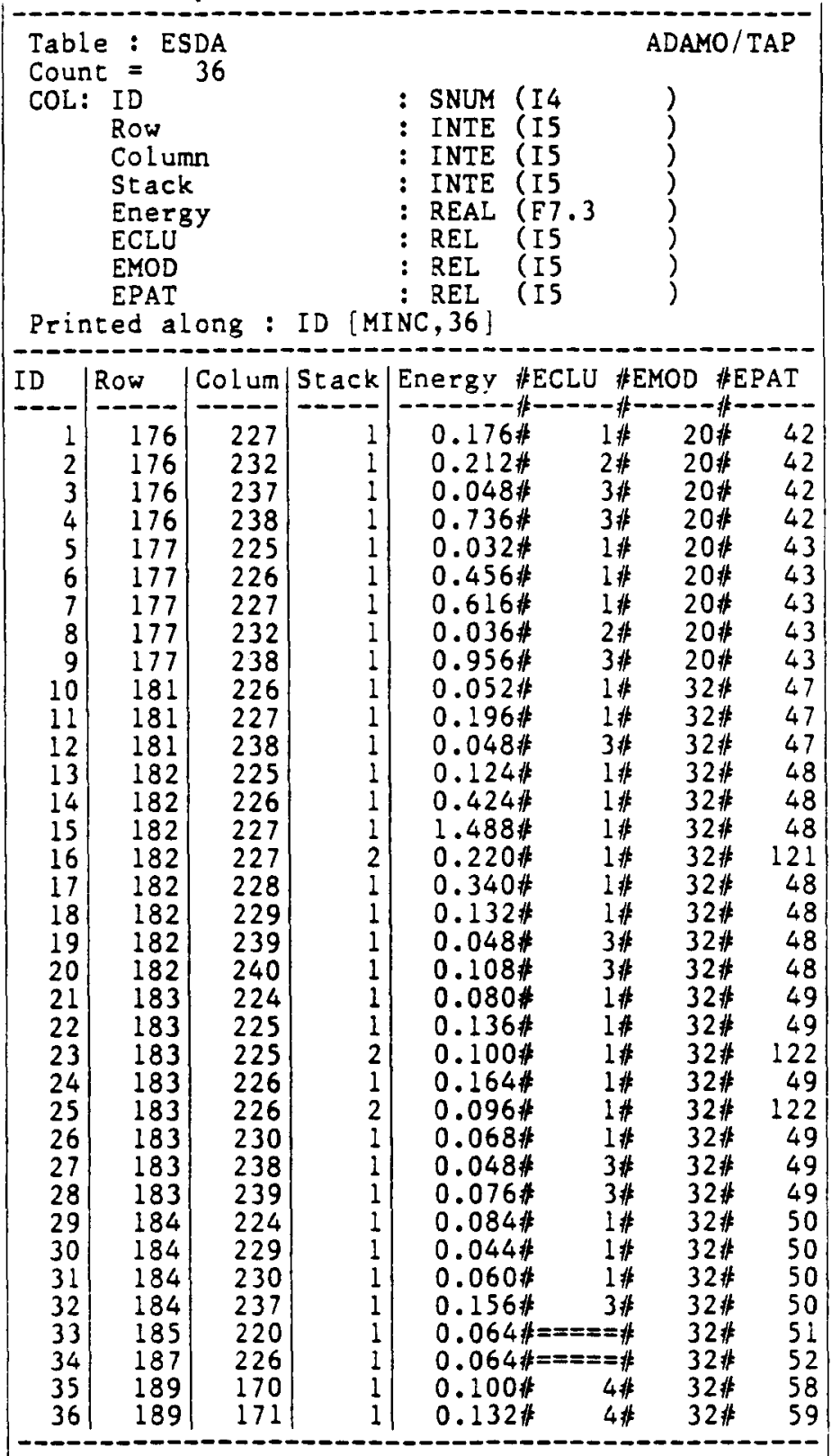

Fig. 6. Tables produced by the ECAL code. 
code would need little restructuring. This is not necessarily the way it would have been done in the absence of such constraints. The ER diagram was produced, and DDL written and processed in the normal way.

The code was then modified to make use of the TAP. The only difficult part of this work was understanding the original non-TAP code. After performing some simple optimisation the execution speed was similar to the original code, but all references to the underlying memory manager had vanished. Special printing routines were no longer needed and intricate code, which referenced variables in the data structures handled by the memory manager, was eliminated, resulting in a program which was shorter and clearer.

\subsection{Detector description}

The database of the ALEPH Detector Description System comprises all information on the detector, such as the geometrical description of the modules and the read-out geometry. Since the software, like the hardware, is developed at differ- ent places and by many people, a well defined framework and explicit rules were needed. It was realised that a modern approach was required to build and maintain a consistent database. The system had to be open ended, since more information will need to be stored in the future and it must be possible to couple to existing RDBs.

Fig. 7 shows part of the description of the read-out geometry for two of the detector components. A more detailed description of the whole system can be found in [13]. DDL was written, and the ADAMO tools were used to provide a consistency check of the model, to perform data validation, as documentation and to guarantee consistency between the application programs and the database. All these points have been badly neglected in the past as tools have not been available.

The data must be available on computers from different manufacturers and at many sites. For this an ADAMO DAF is used, copied to and from an ORACLE database by the TOR. Fast access to the data and distribution of the database is performed via the DAF, so it is not necessary to have

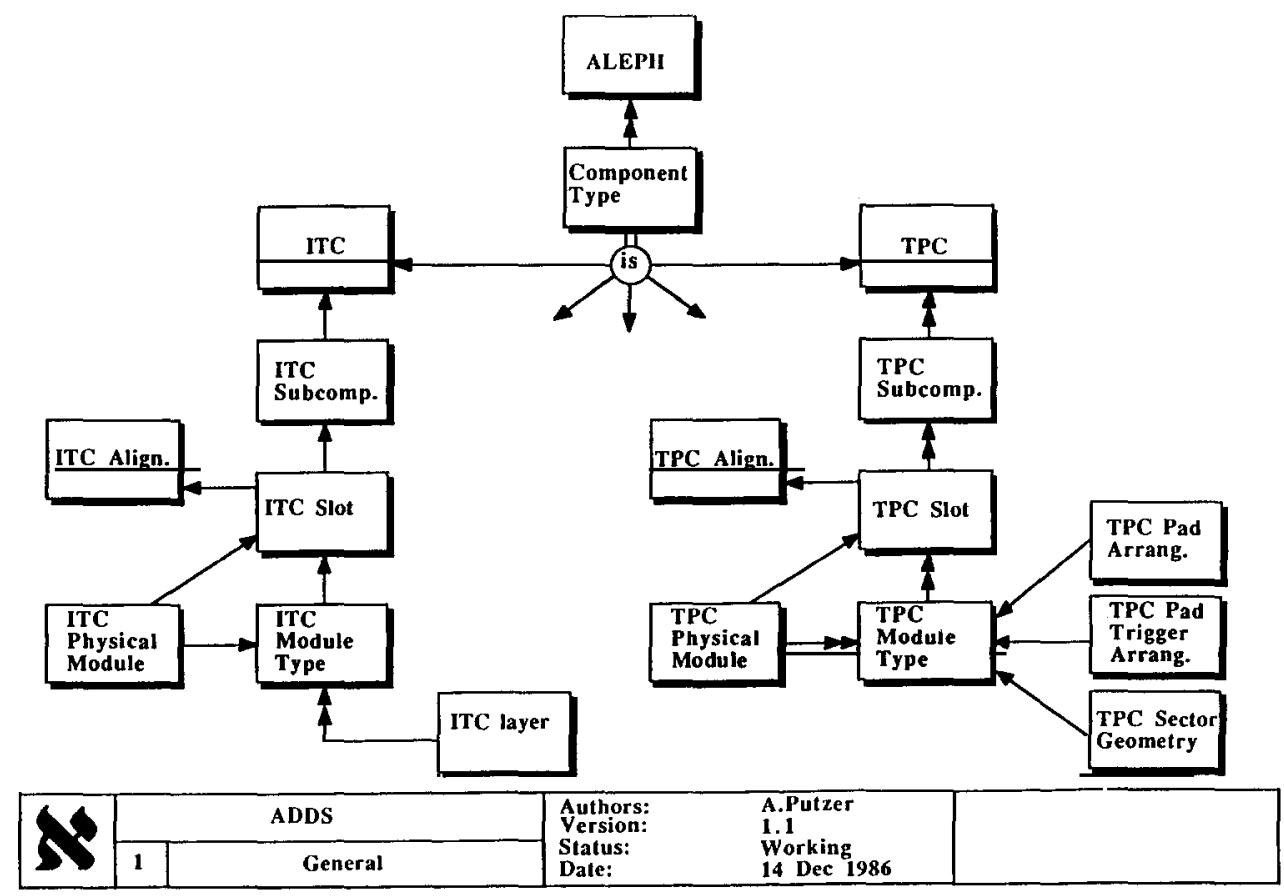

Fig. 7. ER diagram from the Aleph Detector Database System. 
ORACLE running on all installations.

ORACLE maintains the master database, where only people responsible for parts of the detector are allowed to add, change and delete information. Full use may be made of the features provided by the RDBMS and its support tools to enter and maintain some basic constants. The contents of a DAF may be manipulated by a program to enter data such as calibration constants. It is the task of the database administrator to ensure that the master copy (the ORACLE database) is kept up to date.

An additional study on detector description was performed to specify some of the data structures of GEANT 3.07 [14] using the ER model, and to recode some GEANT routines to use the TAP, and to compare with the original [15]. The system was modelled in a way which reflected the existing data structure, to facilitate the recoding of the GEANT routines. The revised routines were shorter and easier to read as there were no longer any references to the memory manager. Certain features of GEANT which were not very clear from the manual, were evident in the ER diagram, and of course the whole data structure was very clearly described by the DDL.

\subsection{Use in an online system}

The complexity of modern HEP experiments poses severe problems for the design of the online systems used to operate the experiments. The arrangement of detector elements is complicated and together they generate several hundred thousand channels of information. A readout system is needed to digitise and record all these data and a control system is required for setting and monitoring the operating state of the equipment [16].

Sophisticated software must be developed to configure the experiment for data taking, for controlling access to all system resources and for managing the large quantity of information generated by the experiment. All this must be done in such a way that the operator is screened from unnecessary complexity. Only with a real understanding of the data and their relationships can such a system be constructed.

The ER model has been found capable of representing the essential online information on diagrams which are clear and easy to interpret $[17,18]$. The scope of the data model used in the design of the ALEPH online system is shown in fig. 8. The Detector Description, explained in the previous section, forms a central reference point in this model. In addition, much effort has been put

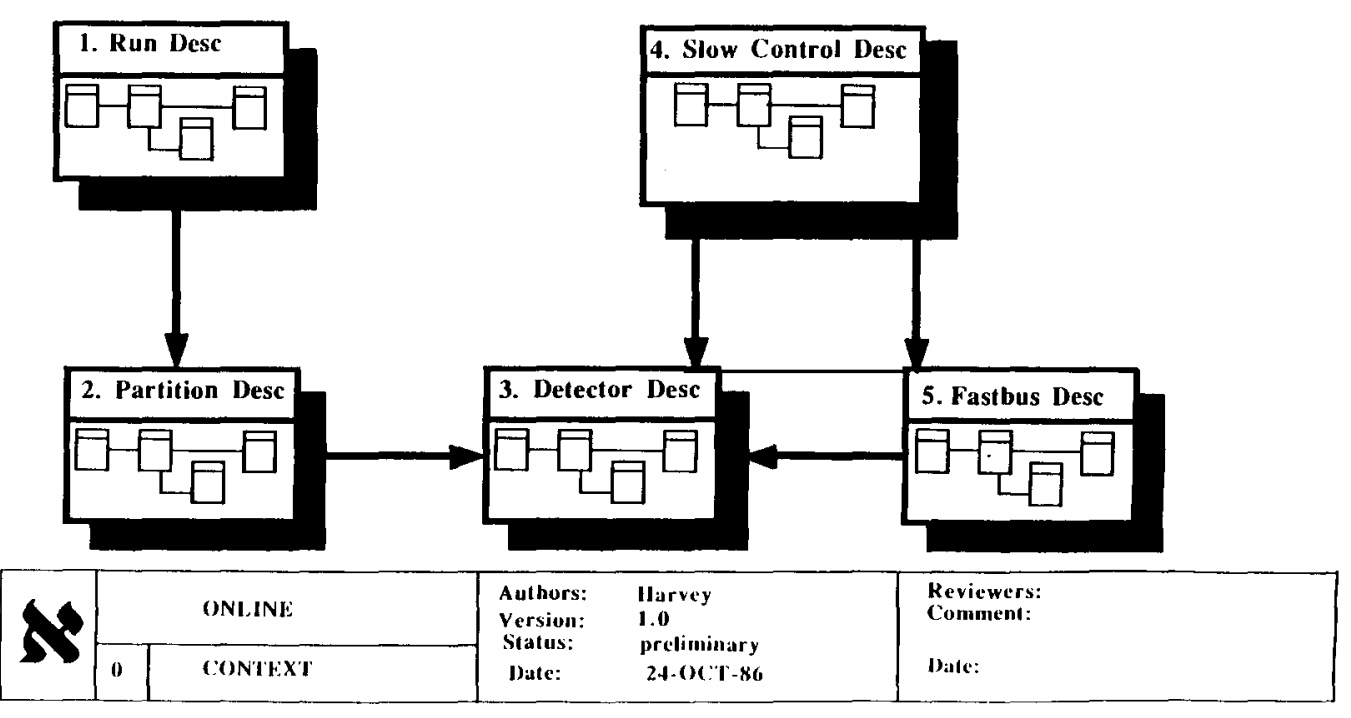

Fig. 8. ER Context diagram of the ALEPH on-line system. 


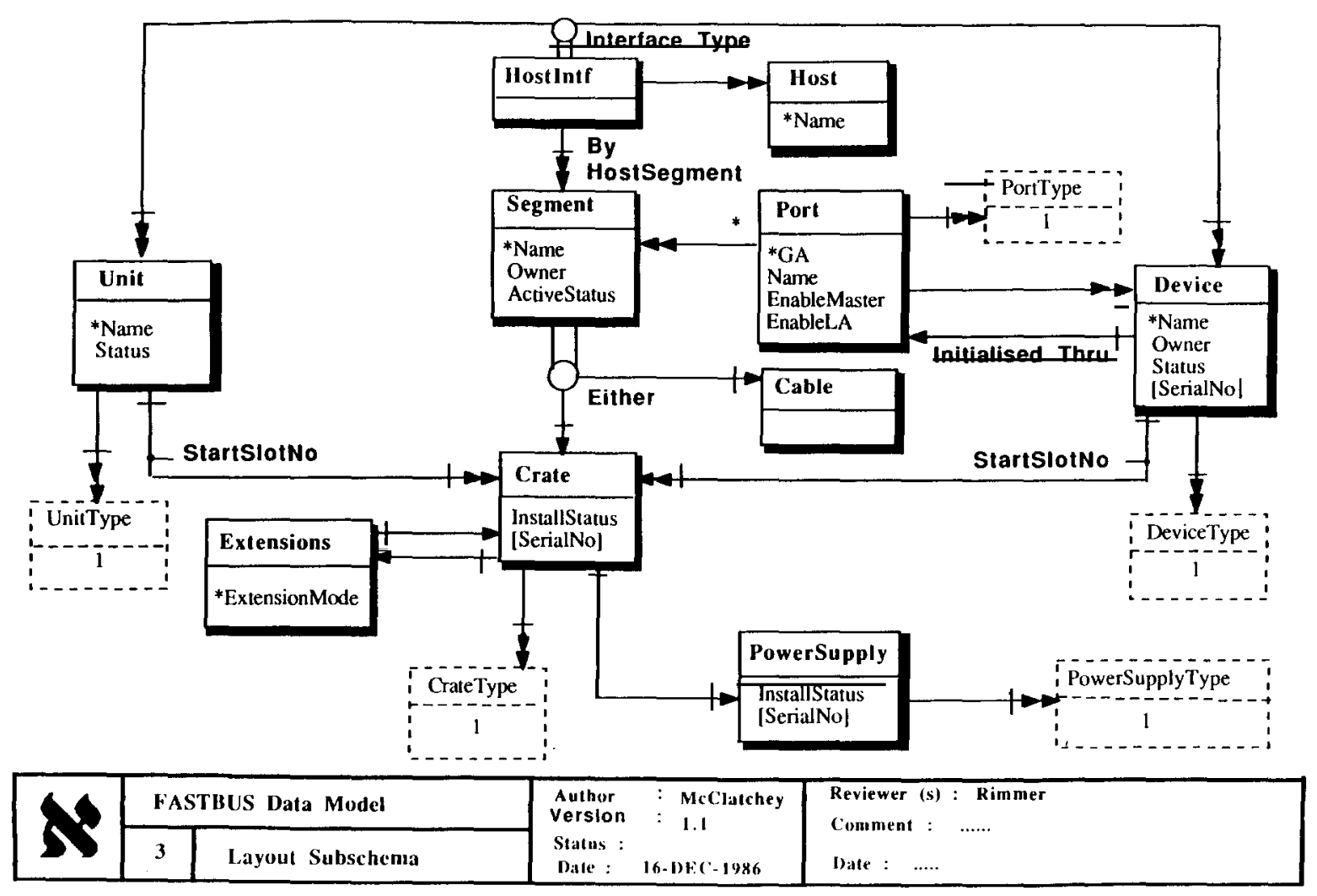

Fig. 9. ER diagram from the FASTBUS description.

into describing our readout system which is based on Fastbus.

Fastbus is a segmented, high speed bus for establishing communication paths between the intelligent processing modulus used to digitise, collect and process raw signals coming from the detectors. The possible ways in which devices can be configured in Fastbus systems is complicated, as are the rules governing the passing of operations between devices. Fig. 9 illustrates this point by an extract from the Fastbus Description showing connectivity and addressability of the readout system. One of the great benefits of following this approach has been that the diagrams have been analysed in great detail to check that they offer a correct interpretation of the Fastbus standard. This gives confidence in the correctness of the program structure developed from the data specification.

The automated initialisation software used to configure a Fastbus system requires access to items stored in the database. These access procedures must allow data to be stored and retrieved from the database and to follow relationships between data items, but should hide the details of data storage. The TAP package of ADAMO provides a set of standard primitives to manipulate ER structures and has been found to be ideal for implementing the access procedures, resulting in clear and concise code. Due to the complexity of the Fastbus model and the range of operations to be carried out on the data, many such procedures must be provided. As the TAP will be used to access data throughout the database it is simple to maintain a general data access package in the online environment. The Fastbus data tables are stored on ADAMO DAFs. As with the detector description system the TOR was used to copy these DAFs to and from an ORACLE database, to allow ADAMO and ORACLE to each do the job they do best. 


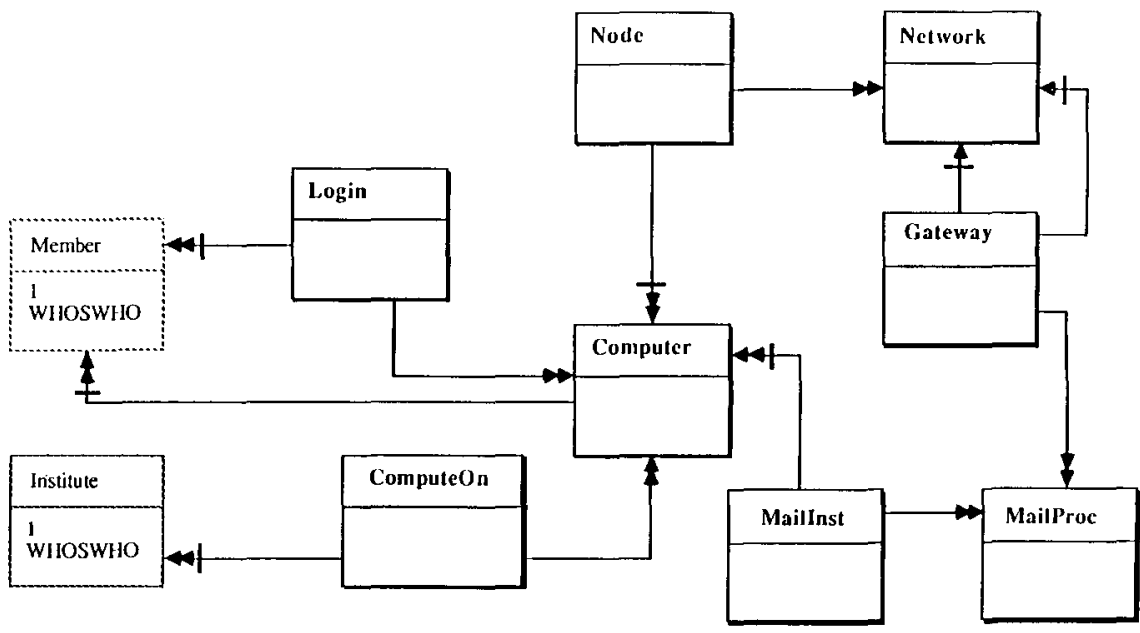

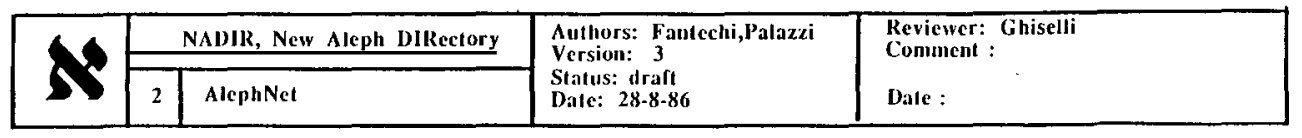

Fig. 10. ER diagram for the New Aleph Directory.

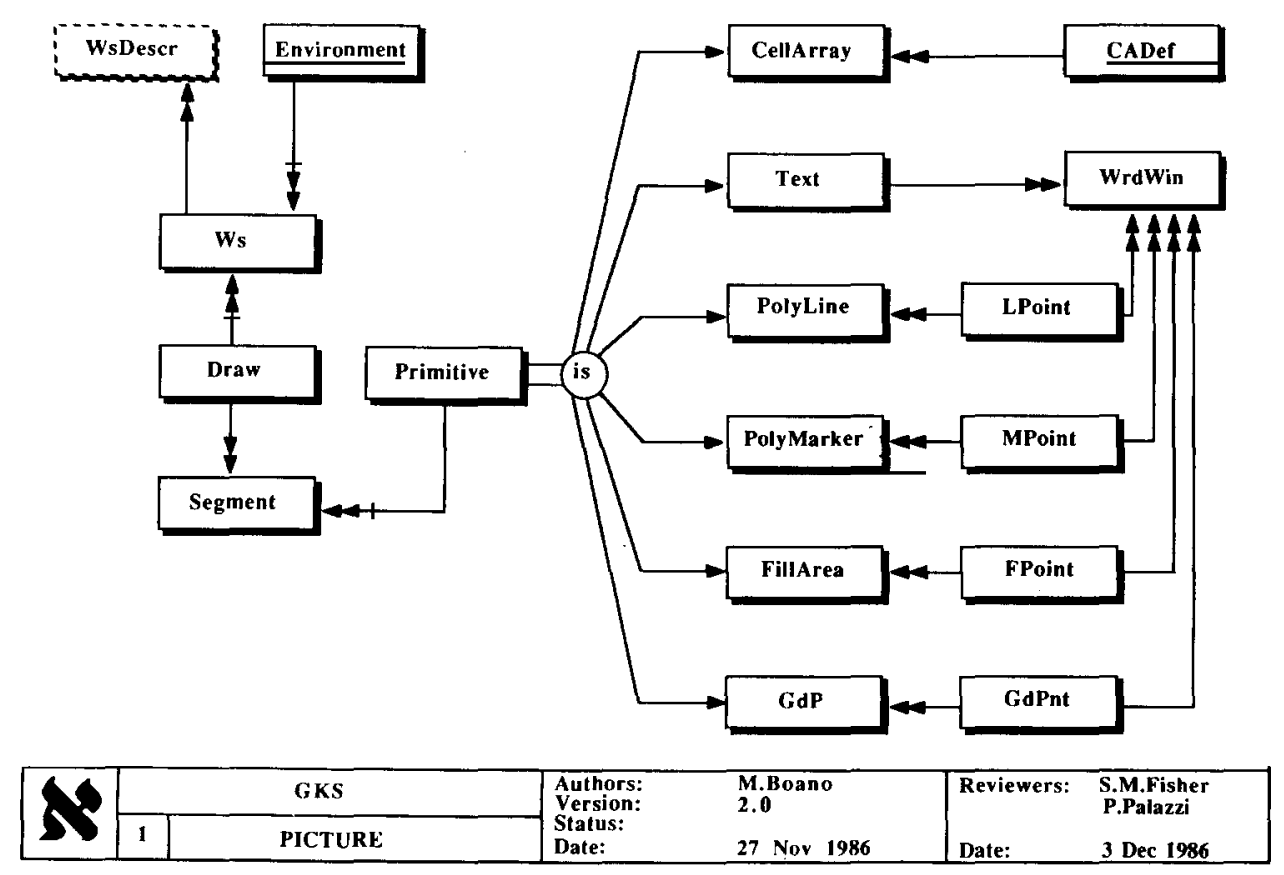

Fig. 11. The 'picture' subschema of GKS. 


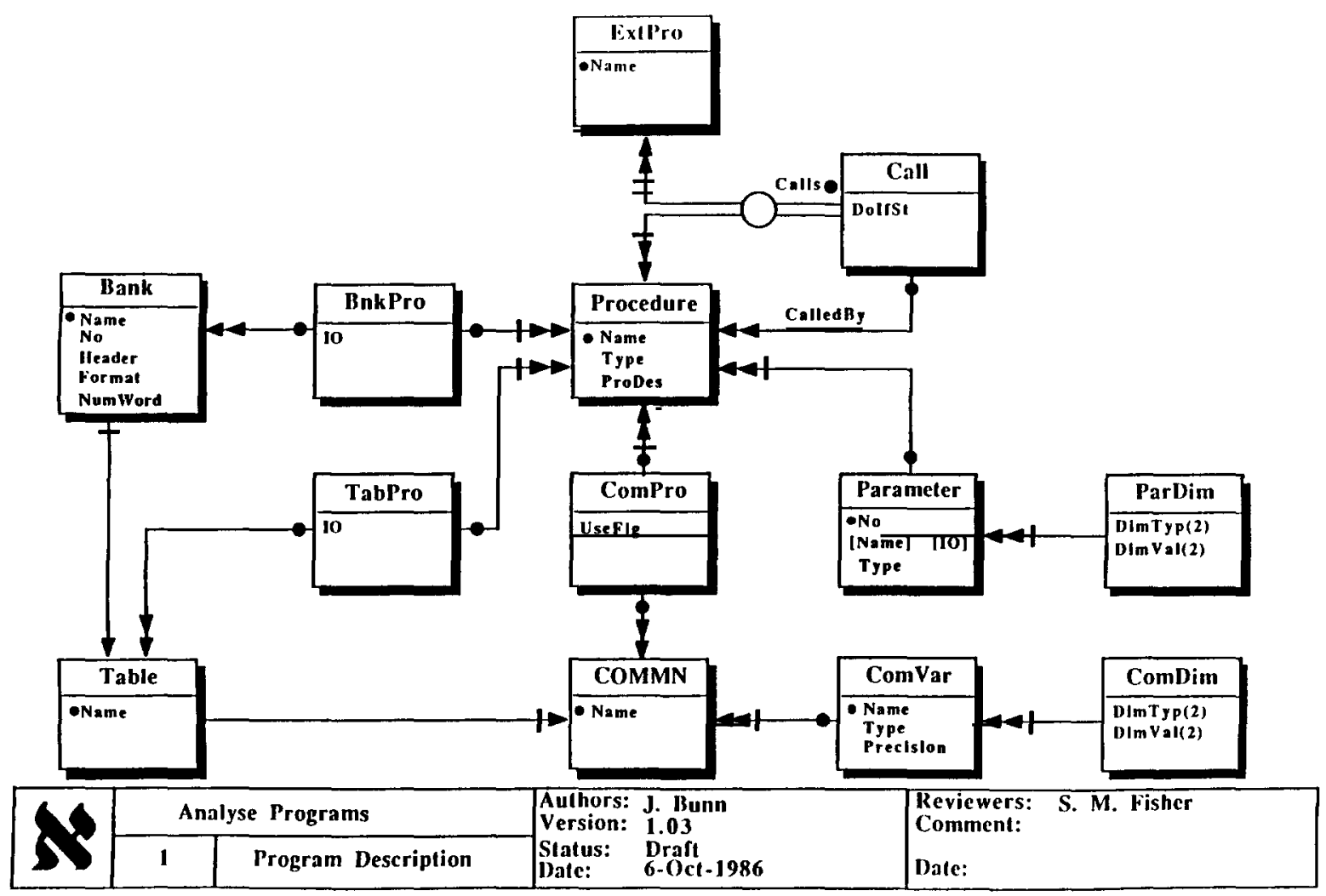

Fig. 12. ER diagram of a FORTRAN program.

\subsection{Experiment directory}

Information exchange is of the utmost importance in a large collaboration. The ALEPH secretariat takes care of mailing papers and technical notes, both within CERN and to outside institutes. The information useful to reach people at CERN, at their Institute or at home is made available to all group members in the form of a printed directory. It contains information such as telephone numbers, addresses, telex and telefax numbers. In the past years all this was managed with WYLBUR EXEC files.

In 1986 the New Aleph DIRectory [19] was built, to take advantage of the availability of the ORACLE RDBMS. Offering initially the same functionality as the old system, it was extended to cover electronic mail and program update distribution. The database was designed with the ER model (fig. 10), with automatic generation by the
ADAMO tools of the commands (SQL) to create the tables and indices in ORACLE.

The interactive updates and queries are carried out via full screen forms provided by the ORACLE support tools. The TOR is used to copy data concerning computers, members, logins, networks and mail servers from the ORACLE database to a DAF. This DAF is read by a program (installed at ALEPH institutes where ORACLE may not be available) which sends electronic mail to ALEPH members using the appropriate systems and gateways [20].

\subsection{GKS}

The Graphical Kernel System (GKS) provides a set of low level functions for graphics programming. The standard (ISO 7942) specifies GKS mostly by words with a very few diagrams to aid understanding. The standard took a long time to produce (1976-1986), and commercial GKS 
packages were also slow to appear. This enormous time span results in obsolescent products. One must move rapidly from first specification to a working system; for this to be possible the specification must be clear enough to allow wide exchange of ideas and there must be a simple way to construct the system from its specification.

The ADAMO techniques were used together to specify a GKS-2D system [21]. For complete GKS-2D only 32 Entity Sets were needed, i.e. it is inherently a simple system, though at first sight, having 201 subroutines, GKS appears complicated.

Fig. 11 show the 'picture' subschema. In GKS, pictures are constructed from six output primitives: polyline, polymarker, text, fill area, cell array and generalized drawing primitive (GDP), each of which has associated with it a set of parameters, for each instance of the primitive. Primitives may be grouped together into units known as segments which are stored, and may be manipulated as, a single object. A segment may contain many graphics primitives (it may also be empty).

Dataflow diagrams were used to describe the overall system and the way in which different classes of subroutine calls are processed, state transition diagrams were used to show the states of the system and algorithms were simply described by means of the final code. It was a straightforward task to build GKS as the TAP allows the programmer to continue to think in terms of entities and relationships, and so follow a simple path from specification to code. Timing tests indicate that the execution speed of our GKS is comparable with the fastest commercial package we could find.

The specifications occupied a junior programmer, unfamiliar with ADAMO, for about 6 weeks. The system (which is at level $2 b$ except for some inquiry and input routines) took about 4 man weeks for the initial coding with one partial device driver. The building of a GKS system in such a short time, from our ER specification, shows clearly the benefits of the method.

\subsection{Software engineering tools with ADAMO}

The ADAMO system was constructed using as far as possible the ADAMO system itself [22]. The use that was made of VMS and the VAXSet in the development and maintenance of the system has been described in ref. [23]. The tools all make full use of ADAMO and are written with the TAP. The TAP itself makes only limited use of TAP calls. It uses a memory manager but has been structured to be well layered such that the memory manager is readily changeable. The system (including tests and documentation) contains about 40000 lines of source code and has, so far, taken about 11 man-years.

Another application in this field is a program to analyse FORTRAN code and produce reports [24]. Fig. 12 shows the ER diagram of a FORTRAN program, which makes use of a memory manager and of the TAP. The input to the program is generated by a customised version of FLOP [25], which is able to parse the FORTRAN. The FLOP file is used to fill tables managed by the TAP. Many possibilities exist for producing report generators which examine these tables. One such generator has been written to show the calling structure of a program. The report should correspond to the structure chart of the program.

\section{Conclusions}

There is general agreement within ALEPH that the Entity-Relationship model of ADAMO and the related tools are very useful in the analysis and design phases of complex software projects. They are now, together with SASD, part of the software development strategy of the experiment, and have provided the analyst/designer with a solid method, as opposed to the heuristic approaches used previously. The decision to use the model and the tools requires that significant effort MUST be spent at the modelling stage leading to a much clearer insight into the structure of the data before coding begins. This greatly increases the probability of producing the desired system. Modelling existing complex systems, such as Fastbus or GKS, makes them easy to understand.

Experience in coding from scratch with the TAP, as well as in recoding existing programs proves that coding and debugging time is much shorter than with the traditional style of work. 
The code produced is shorter (much shorter if it involves sorting), uses meaningful variable names and is therefore much more readable and maintainable. The TAP routines access the data structures by copying rows of tables to and from COMMON blocks, and provide checks which frequently catch bugs during program development. An initialisation routine is produced automatically, as are COMMON and type declaration statements. In this way, the application code is as invariant to changes in the data structures as it can be (just recompilation and relinking is required), and it cannot be inconsistent with the data, nor with their description.

Accessing data through calls to the TAP is necessarily a little slower than addressing data elements by an offset in a vector, or using FORTRAN arrays with no memory management package. Substantial gains in speed are generally obtained by finding better algorithms. Since using ADAMO gives a better design, allows faster coding, and does not obscure the algorithm in intricate code, a faster program may be obtained for the same effort.

\section{Acknowledgements}

Takis Kokkinias and Stratos Zevgolatakos wrote the prototypes of the ORACLE interface tools.

We are indebted to Sau Lan Wu, Friedrich Dydak, Mike Metcalf, John Thompson, Klaus Tittel and Weimin Wu for their support.

We profited much from discussions with Gottfried Kellner, Jurgen Knobloch, Jean-Francois Renardy and Henri Videau.

\section{References}

[1] D.C. Tsichritzis and A. Klug, The ANSI/X3/SPARC DBMS framework report of the study group on database management systems, Information Systems, vol. 3 (1978).

[2] D.C. Tsichritzis and F.H. Lochovsky, Data Models (Prentice-Hall, Englewood Cliffs, 1982).
[3] E.F. Codd, 1981 Turing Award Lecture.

[4] P.P. Chen, ACM Trans. on Database Systems 1 (1976) 9.

[5] R. Brazioli, S.M. Fisher, P. Palazzi and W. Zhao, The architecture of the ADAMO system, ADAMO note 4, version 2.2 (November 1986).

[6] S.M. Fisher, An example of the application of ADAMO methods and tools to a problem, ADAMO note 2, version 2.2 .

[7] T. DeMarco, Structured Analysis and System Specification (YOURDON Press, 1978).

[8] ORACLE, Oracle Corporation, California, USA

[9] G. Kellner, Comput. Phys. Commun. 45 (1987) A21.

[10] M.G. Green, JULIA/ECAL, cluster finding and track matching in the ALEPH reconstruction program, ADAMO Application Note 4.

[11] The ADAMO application notes, ADAMO Application Note 0 (Nov. 1986).

[12] S.M. Fisher, GALEPH/ITC, rewrite simulation of ITC using the ADAMO/TAP, ADAMO Application Note 6.

[13] A. Putzer, ADDS, The Aleph Detector Description System, ADAMO Application Note 12 (in preparation).

[14] R. Brun, F. Bruyan, M. Maire, A.C. Pherson and P. Zanarini, GEANT3 User's Guide, CERN DD/EE/84-1 (May 1986).

[15] S.M. Fisher, GEANT (3.07) Volume, material and media data structure, ADAMO Application Note 1.

[16] T. Charity, J. Harvey and R. McClatchey, Comput. Phys. Commun. 45 (1987) 433.

[17] R. McClatchey, FASTBUS Initialize/Verify, ADAMO Application Note 5.

[18] J. Harvey, S. Tang and W. Zhao, Manage PARTITIONS in the ALEPH data acquisition system, ADAMO Application Note 9.

[19] F. Blin, S.M. Fisher, W.G. Moorhead, P. Palazzi and Z. Qian, NADIR, the New Aleph DIRectory, ADAMO Application Note 10.

[20] V. Emiliani, R. Fantechi, P. Palazzi and Z. Qian, AMSEND, a utility to send electronic mail within ALEPH, ADAMO Application Note 11.

[21] M. Boano, R. Brazioli, S.M. Fisher, P. Palazzi and W.R. Zhao, Specifying and Building Graphics Systems with Entity-Relationship, Dataflow and State Transition Diagrams: GKS-2D, submitted to Eurographics '87.

[22] R. Brazioli, S.M. Fisher, P. Palazzi and W. Zhao, Using ADAMO to design and build ADAMO, ADAMO Application Note 8 (Nov. 1986).

[23] R. Brazioli, S.M. Fisher, P. Palazzi and W. Zhao, Use of VMS and the VAXSet for the ADAMO Data Management System, 1986 DECUS Symposium Hamburg, September 1986 and $\mathrm{DD} / 86 / 16$.

[24] J. Bunn, PRODES, a tool to analyze and describe a FORTRAN program, ADAMO Application Note 3.

[25] H. Grote, FLOP, CERN DD/US/13. 